\title{
Spontaneous intracerebral hemorrhage during left hemihepatectomy in a cirrhotic patient
}

\author{
Chang Woo Lim, Hui Young Kim, Hyung-Joo Chung \\ Department of Anesthesia and Pain Medicine, Kosin University College of Medicine, Busan, Korea
}

Received February 25, 2019

Revised March 14, 2019

Accepted April 15, 2019

Corresponding author

Hyung-Joo Chung

Department of Anesthesia and Pain

Medicine, Kosin University College

of Medicine, 262, Gamcheon-ro,

Seogu, Busan 49267, Korea

Tel: +82-51-990-6474

Fax: +82-51-254-2504

E-mail:dmadjejs@naver.com

ORCID:

https://orcid.org/0000-0001-9545-3245

\begin{abstract}
Spontaneous intracerebral hemorrhage $(\mathrm{sICH})$ is bleeding that occurs without trauma or known structural abnormality. Hypertension is a main cause of sICH, but liver cirrhosis may be another reason; however, this rarely occurs under anesthesia. We report on a case of sICH in a 75-yearold woman patient with liver cirrhosis during left hemihepatectomy. And the literature on the usefulness of perioperative bispectral index monitoring for early detection of brain damage is reviewed.
\end{abstract}

Keywords: Spontaneous intracerebral hemorrhage; Liver cirrhosis; Hemihepatectomy; Bispectral index

\section{INTRODUCTION}

An intracerebral hemorrhage (ICH) is an intraparenchymal bleed. The incidence rate in the United States is about 12-15 per 100,000 [1].

Spontaneous ICH (SICH) is bleeding that occurs without trauma or known structural abnormality [2]. Risk factors include hypertension, age $>85$ years, an anticoagulant medication, trauma, history of previous stroke or $\mathrm{ICH}$, chronic renal disease, aneurysm or vascular malformation, abuse of alcohol or drugs [2], and liver cirrhosis [3]. Symptoms can manifest as dysfunction when the lesions are pressurized by hemorrhage, and intracranial pressure can rise due to a mass effect. Loss of consciousness or ear bleeding may occur, leading to a coma before the bleed is identified.
Computed tomography angiography (CTA) or magnetic resonance angiography (MRA) can confirm the diagnosis [4].

Unfortunately, anesthetized and unconscious patients cannot complain of symptoms. It is difficult to perform CTA or MRA to diagnose ICH, and even if found, hemorrhage is likely to be detected only after significant damage. The authors want to share our experience of SICH and show the usefulness of the bispectral index (BIS) monitoring for detecting brain damage under anesthesia.

\section{CASE REPORT}

A 75-year-old woman diagnosed with hepatocellular carcinoma and Child-Pugh class A liver cirrhosis was hospitalized for partial hepatectomy. She was diagnosed with 
hepatitis B virus infection in 1960 and was not an alcohol consumer. Hepatocellular carcinoma was diagnosed in December 2011, with a single radiofrequency ablation in 2012 and 3 trials of transarterial chemoembolization between 2014 and 2016.

Bronchiectasis was seen on preoperative X-ray, and no abnormal findings were seen on other examinations, except for a tachyarrhythmia on the electrocardiogram (ECG). Prothrombin time $(13.1 \mathrm{sec})$ and PT INR (1.03) were the normal range but platelet count was decreased $(116,000 / \mu \mathrm{L})$.

When the patient arrived at the operating room, we initiated noninvasive blood pressure (NIBP), pulse oximetry, ECG, and BIS monitoring. Anesthesia was induced with propofol $60 \mathrm{mg}$, rocuronium $50 \mathrm{mg}$, mobinul $0.2 \mathrm{mg}$, and remifentanil $60 \mu \mathrm{g}$, after which the patient was intubated. At that point, the NIBP was $150 / 60 \mathrm{mmHg}$ and the BIS score was 63. This blood pressure was the highest blood pressure for this operation. Anesthesia was maintained with $1.5 \%$ sevoflurane and $800 \mu \mathrm{g} /$ hour remifentanil, with a tidal volume of $400 \mathrm{~mL}$ at 12 breaths per minute. After that, the left radial artery was cannulated. The central venous catheter was inserted into the right subclavian vein and the operation was started. When the liver was dissected and resection was started, the BIS score dropped to 25 and the blood pressure measured on the arterial line was decreasing. Two doses of $50 \mu \mathrm{g}$ phenylephrine were given, but the blood pressure was not stable and a continuous infusion was started at $3 \mu \mathrm{g} / \mathrm{kg} /$ minute. The BIS score also changed with the blood pressure. Bleeding occurred at the time of hepatectomy The BIS score
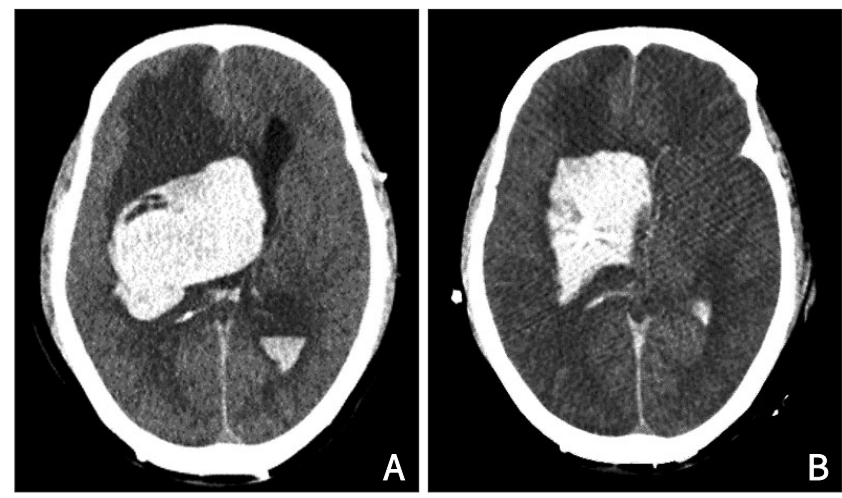

Fig. 1. Brain CT finding (A) after left hemihepatectomy and (B) after external ventricular drainage with hematoma aspiration.
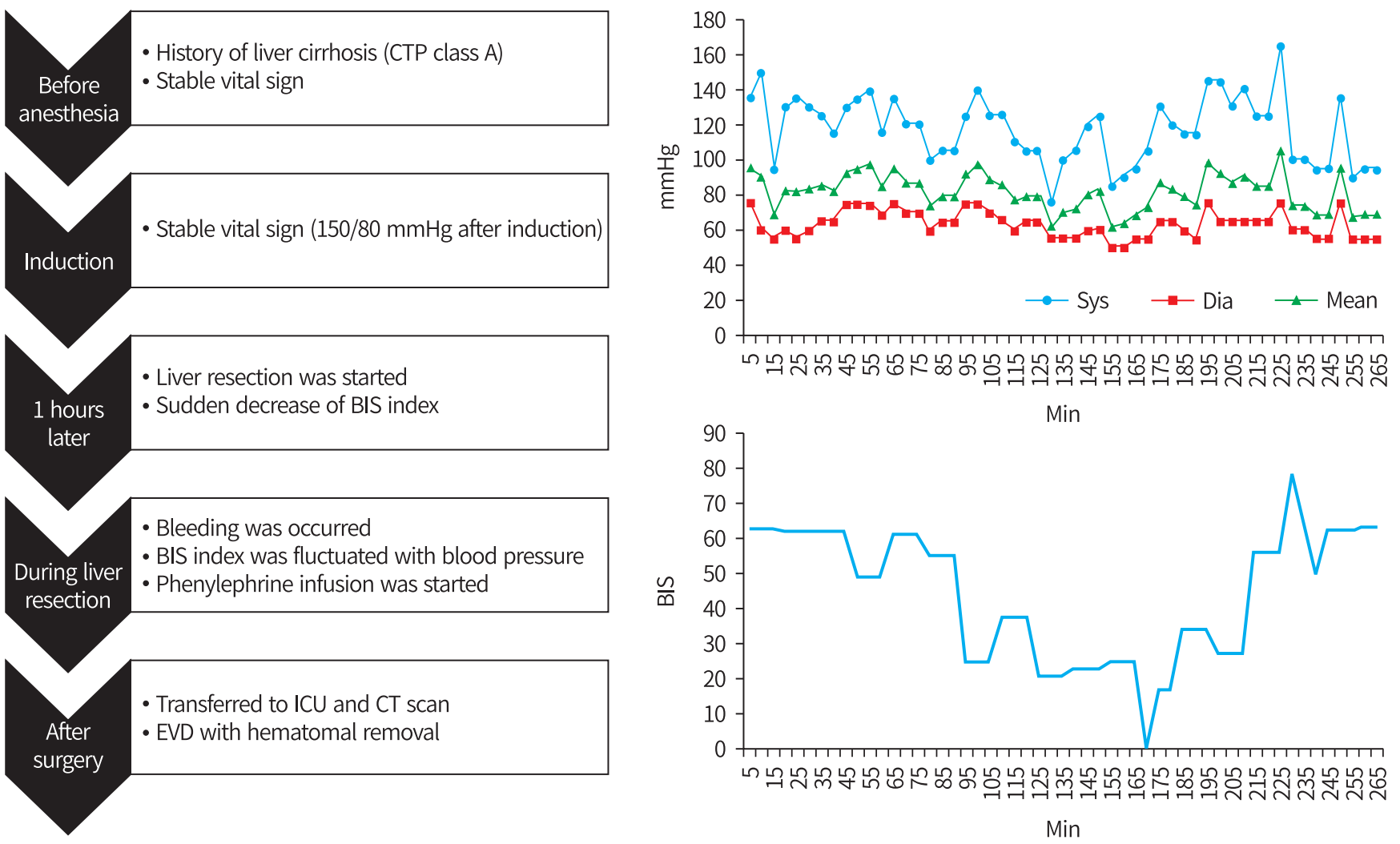

Fig. 2. Timeline and vital trend during operation. BIS: bispectral index, ICU: intensive care unit, CT: computed tomography, EVD: external ventricular drainage. 
further decreased to 0 at that time and we suspect the brain damage. The BIS score increased to 20 when the infusion of phenylephrine was increased to $7 \mu \mathrm{g} / \mathrm{kg} / \mathrm{min}$. Blood loss was measured at about 1,200 mL, so $2,000 \mathrm{~mL}$ of crystalloid was injected and two units of packed red blood cells were transfused. Thereafter, the BIS score changed along with blood pressure and the operation proceeded. At the end of the surgery, sevoflurane was stopped and 200 mg of sugammadex was administered. However, the BIS score did not increase above 70 . The patient did not recover consciousness and was no response to any stimulation. We judged that she was a comatose state, so she was transferred to the intensive care unit with intubation. ICH was found on computed tomography (CT) and neurosurgery was performed for external ventricular drainage (EVD) with hematoma aspiration (Fig. 1), but she expired 7 days after surgery. Information from this case is described in a timeline (Fig. 2).

\section{DISCUSSION}

Patients with liver cirrhosis reportedly have a higher incidence of intracerebral hemorrhage, however, there is no significant correlation to subarachnoid hemorrhage or subdural hemorrhage [5]. SICH occurs in only $0.7 \%$ to $0.8 \%$ of patients with liver cirrhosis [6] but is associated with high mortality and disability rates among survivors. Liver cirrhosis-related SICH is associated with acquired hemostatic deficiency, including thrombocytopenia and coagulopathy [5]. But thrombocytopenia does not always require platelet transfusion. Hypertension was less frequent in patients with chronic liver disease and liver cirrhosis [7]. This may suggest that patients with liver dysfunction are more vulnerable to developing ICH than those without such background pathology. The volume of the bleed effects prognosis, but patients under anesthesia are unconscious and cannot report symptoms If SICH is suspected, CT is the first step [8]. However, if surgery is underway, it may be difficult to perform. Therefore, careful observation by the anesthesiologist during surgery is required in patients with risk factors for SICH.

BIS monitoring is a simple, noninvasive, and easy-to-understand tool that analyzes the electroencephalogram of a patient during surgery and is represented by a number between 0 and 100 [9]. Intensive care unit (ICU) protocols have been developed to monitor the depth of sedation and the degree of awareness of patients during general anesthesia [10]. BIS is currently being used to monitor the state of consciousness of trauma patients in ICUs [11]. A BIS values of 0 and suppression ratio (SR) of 100 was reportedly able to assist in the early detection of brain death in 19 patients as an additional test [0]. Another report indicated that transcranial Doppler was associated with cerebral circulation arrest when the BIS score was 0 and the SR was 100 [12].

The BIS score and Glasgow Coma Score (GCS) were found to be correlated in the assessment of head trauma patients in the ICU [13]. Another study reported a BIS score of 96.2 when the GCS corresponded to mild head injury, a BIS score of 45.5 when the GCS corresponded to moderate head injury, and a BIS score of 31.3 when the GCS corresponded to severe head injury [14].

In our case, BIS score suddenly dropped from $50^{\text {th }}$ to $20^{\text {th }}$ level with no event during general anesthesia, and BIS score was temporarily found 0 with an SR of 100 , so we suspected brain damage and recommend radiologic examination to surgeons. Immediately after surgery, SICH was diagnosed by CT. Although the BIS does not monitor the brainstem directly, it monitors the outside of the brainstem regionally and can be used for earlier detection of brain herniation or worsening cerebral activity [15].

In conclusion, patients with liver cirrhosis need to keep in mind the occurrence of SICH because there is no additional underlying disease at the time of surgery, so close monitoring of the anesthesiologist is necessary for early detecting symptoms due to SICH during anesthesia. If there is a sudden decrease in the BIS score without any special event during surgery, the brain damage may be suspected.

\section{CONFLICT OF INTEREST}

No potential conflict of interest relevant to this article was reported.

\section{REFERENCES}

1. Aguilar MI, Demaerschalk BM. Intracerebral hemorrhage. Semin Neurol 2007;27:376-84.

2. Aguilar MI, Freeman WD. Spontaneous intracerebral hemorrhage. Semin Neurol 2010;30:555-64. 
3. Kandasamy R, Tharakan J, Idris Z, Abdullah JM. Intracranial bleeding following induction of anesthesia in a patient undergoing elective surgery for refractory epilepsy. Surg Neurol Int 2013;4:124.

4. Yeung R, Ahmad T, Aviv RI, de Tilly LN, Fox AJ, Symons SP. Comparison of CTA to DSA in determining the etiology of spontaneous ICH. Can J Neurol Sci 2009;36:176-80.

5. Huang HH, Lin HH, Shih YL, Chen PJ, Chang WK, Chu HC, et al. Spontaneous intracranial hemorrhage in cirrhotic patients. Clin Neurol Neurosurg 2008;110:253-8.

6. Chen CC, Huang YC, Yeh CN. Neurosurgical procedures in patients with liver cirrhosis: a review. World J Hepatol 2015;7:2352-7.

7. Hoya K, Tanaka Y, Uchida T, Takano I, Nagaishi M, Kowata K, et al. Intracerebral hemorrhage in patients with chronic liver disease. Neurol Med Chir (Tokyo) 2012;52:181-5.

8. Broderick JP, Adams HP Jr, Barsan W, Feinberg W, Feldmann E, Grotta J, et al. Guidelines for the management of spontaneous intracerebral hemorrhage: a statement for healthcare professionals from a special writing group of the Stroke Council, American Heart Association. Stroke 1999;30:90515.

9. Escudero D, Otero J, Muñiz G, Gonzalo JA, Calleja C, González A, et al. The bispectral index scale: its use in the detection of brain death. Transplant Proc 2005;37:3661-3.

10. De Deyne C, Struys M, Decruyenaere J, Creupelandt J, Hoste E, Colardyn F. Use of continuous bispectral EEG monitoring to assess depth of sedation in ICU patients. Intensive Care Med 1998;24:1294-8.

11. Fàbregas N, Gambús PL, Valero R, Carrero EJ, Salvador L, Zavala $\mathrm{E}$, et al. Can bispectral index monitoring predict recovery of consciousness in patients with severe brain injury? Anesthesiology 2004;101:43-51.

12. Stammet P, Werer C, Mertens L, Lorang C, Hemmer M. Bispectral index (BIS) helps predicting bad neurological outcome in comatose survivors after cardiac arrest and induced therapeutic hypothermia. Resuscitation 2009;80:437-42.

13. Paul DB, Umamaheswara Rao GS. Correlation of bispectral index with glasgow coma score in mild and moderate head injuries. J Clin Monit Comput 2006;20:399-404.

14. Ebtehaj M, Yaqubi S, Seddighi AS, Seddighi A, Yazdi Z. Correlation between BIS and GCS in patients suffering from head injury. Ir J Med Sci 2012;181:77-80.

15. Fyntanidou B, Grosomanidis V, Aidoni Z, Thoma G, Giakoumis M, Kiurzieva E, et al. Bispectral Index Scale variations in patients diagnosed with brain death. Transplant Proc 2012;44:2702-5. 